\title{
Triple Coronary Artery Thrombosis Presenting as Acute Anterior ST-Segment Elevation Myocardial Infarction
}

\author{
Emrah Ermis, MD ${ }^{1}$ Serkan Kahraman, MD ${ }^{2}$ Hakan Ucar, MD ${ }^{1} \quad$ Sinem Ozbay Ozyılmaz, MD ${ }^{1}$ \\ Samir Allahverdiyev, $\mathrm{MD}^{1}$
}

${ }^{1}$ Department of Cardiology, Faculty of Medicine, Biruni University, Istanbul, Turkey

${ }^{2}$ Department of Cardiology, Silivri State Hospital, Istanbul, Turkey

Address for correspondence Emrah Ermis, MD, Department of Cardiology, Faculty of Medicine, Biruni University, Eski Londra Asfaltı, No:10, Florya, 34295 Istanbul, Turkey (e-mail: eermis@biruni.edu.tr).

Int J Angiol 2018;27:223-226.

\begin{abstract}
Keywords

- multivessel coronary artery disease

- artery thrombosis

- percutaneous coronary intervention

- flower petal technique

- acute coronary syndrome

- coronary atherosclerosis

- coronary stent

Simultaneous multivessel epicardial coronary artery thrombosis is an uncommon finding in acute ST-segment elevation myocardial infarction (STEMI). It generally leads to cardiogenic shock and sudden cardiac death in the hospital. We report a 42-year-old male patient presenting with acute anterior STEMI with triple coronary artery thrombosis. An emergency coronary angiogram showed total occlusion of the left anterior descending artery ( $L A D)$ with thrombus formation. At the same time, thrombus formations were also seen in the circumflex artery (CXA), the second obtuse marginal (OM2) branch, and the distal right coronary artery (RCA). We unsuccessfully attempted thrombus aspiration of the LAD. Subsequently, we decided to stent the $L A D$, and a successful percutaneous coronary intervention $(\mathrm{PCI})$ was performed for the LAD. In a second procedure, RCA thrombosis regressed with 24-hour tirofiban (glycoprotein Ilb/IIla receptor inhibitor) perfusion, although CXA thrombosis and OM thrombosis did not regress. Therefore, we performed stenting of the CXA and OM with a newer provisional technique called the flower petal technique. Thrombolysis in myocardial infarction (TIMI) flow grade III was seen after stenting. The patient was discharged from the hospital 5 days after $\mathrm{PCl}$ without any symptoms.
\end{abstract}

The typical pathogenetic mechanism of acute myocardial infarction (AMI) is disruption of atheromatous coronary plaque that causes partial or total occlusion of coronary arteries with thrombus formation. Although multivessel coronary artery disease is commonly seen in ST-segment elevation myocardial infarction (STEMI), more than one coronary artery thrombosis is still an uncommon finding. ${ }^{1,2}$ Concomitant ruptured plaques with thrombus formation in all epicardial coronary arteries are a rare angiography finding in STEMI. These patients generally present with cardiogenic shock or sudden cardiac death without percutaneous coronary intervention (PCI). We herein present a case of acute anterior STEMI with triple coronary artery thrombosis.

\section{Case Report}

A 42-year-old male patient was admitted to our emergency service with retrosternal chest pain that had been ongoing for 6 hours. His hemodynamic parameters showed a regular heart rate of 88 beats/minute and blood pressure of 110/80 mm Hg. He had no pathologic properties on physical examination. Electrocardiography (ECG) indicated normal sinus rhythm and ST-segment elevation in both V2-V6 and D2-D3 derivations (-Fig. 1). Coronary angiography was performed immediately due to STEMI. Subsequently, the left main coronary artery (LMCA) was selectively cannulated with a Judkins Left (JL) 4-6F diagnostic catheter (Medtronic, New York, NY). It was shown that the left anterior descending artery (LAD) was totally 


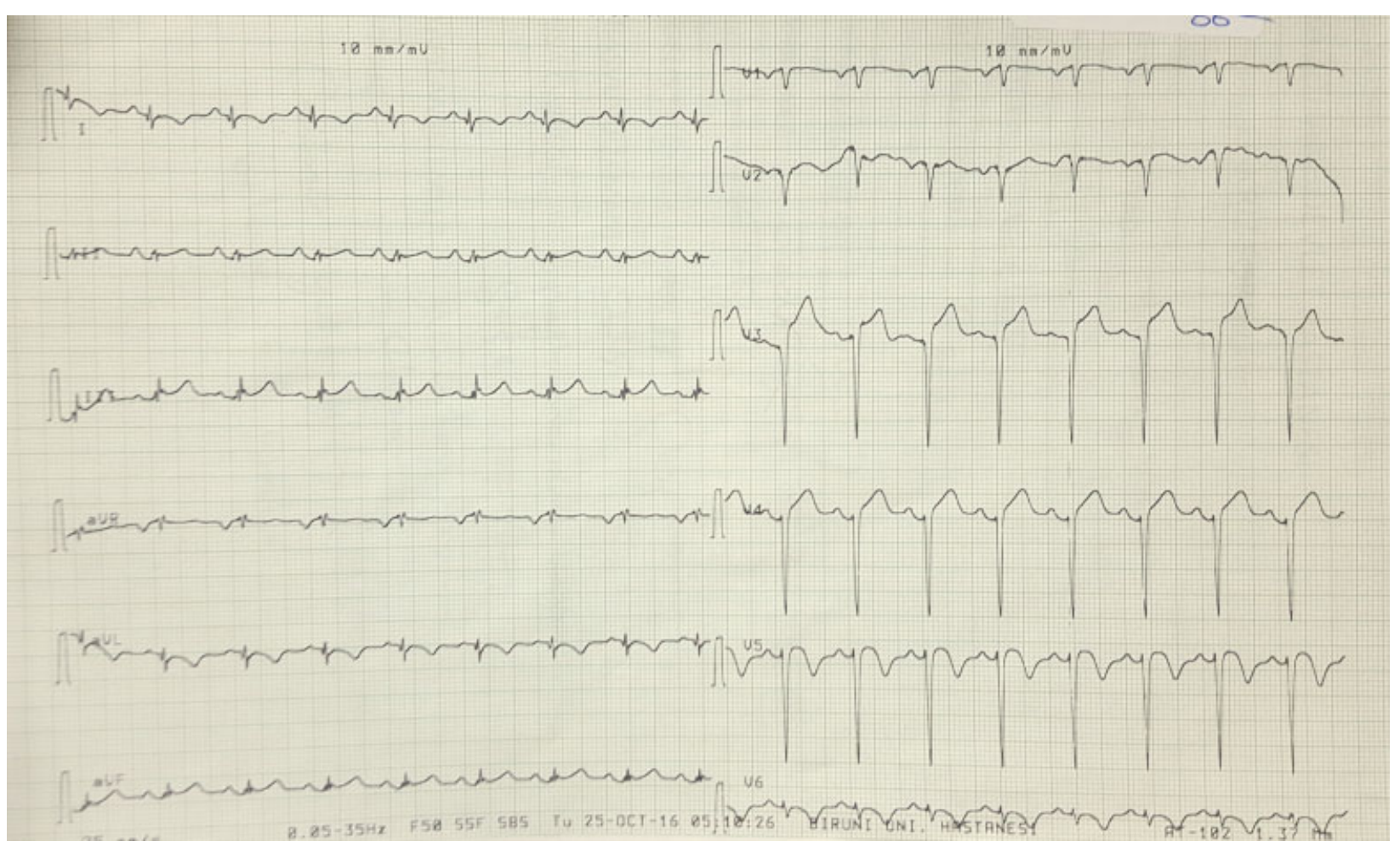

Fig. 1 The patient's electrocardiography (ECG) at the emergency service.

occluded at the end of the proximal segment with thrombus formation. At the same time, additional thrombus formations were seen in the mid-circumflex artery (CXA) and the second obtuse marginal (OM2) branch ( - Fig. 2A). The right coronary artery (RCA) was then selectively cannulated with a Judkins Right (JR) 4-6F diagnostic catheter. It showed partial occlusion with thrombus formation of both the posterolateral(PL) and the

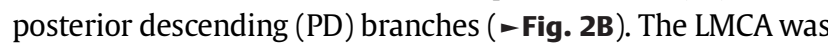
selectively cannulated with a JL 4-6F guiding catheter to perform PCI of the LAD artery due to acute anterior STEMI. Intracoronary thrombus aspiration was first attempted with an intracoronary thrombus aspiration catheter (St. Jude Medical, Inc., St. Paul, MN), but this failed. Therefore, a $3.0 \times 28 \mathrm{~mm}$ drug eluting stent (DES) (Sirolimus eluting coronary stent) was implanted with 18 atm in the LAD (-Fig. 2C). After the stenting procedure was completed, thrombolysis in myocardial infarction (TIMI) flow grade coronary flow improved to TIMI III with good coronary brushing (-Fig. 2D). After LAD stenting, the patient's chest pain was relieved. An intracoronary loading dose and a postloading dose infusion of tirofiban (glycoprotein IIb/IIIa receptor inhibitor) was then administered according to the patient's body weight due to other coronary thrombus formations. After 24-hour tirofiban administration, coronary angiography was performed for the second time. Thrombus formation in the CXA and OM was ongoing, unlike the distal RCA ( - Fig. 3A, B). Therefore, the distal CXA was predilated with a $3.0 \times 15 \mathrm{~mm}$ semicompliant balloon and a $3.0 \times 18 \mathrm{~mm}$ DES (Biolimus eluting coronary stent) was implanted with 16 atm using a modified flower petal technique (-Fig. 3C, D). A second $3.0 \times 18$ mm DES (Biolimus
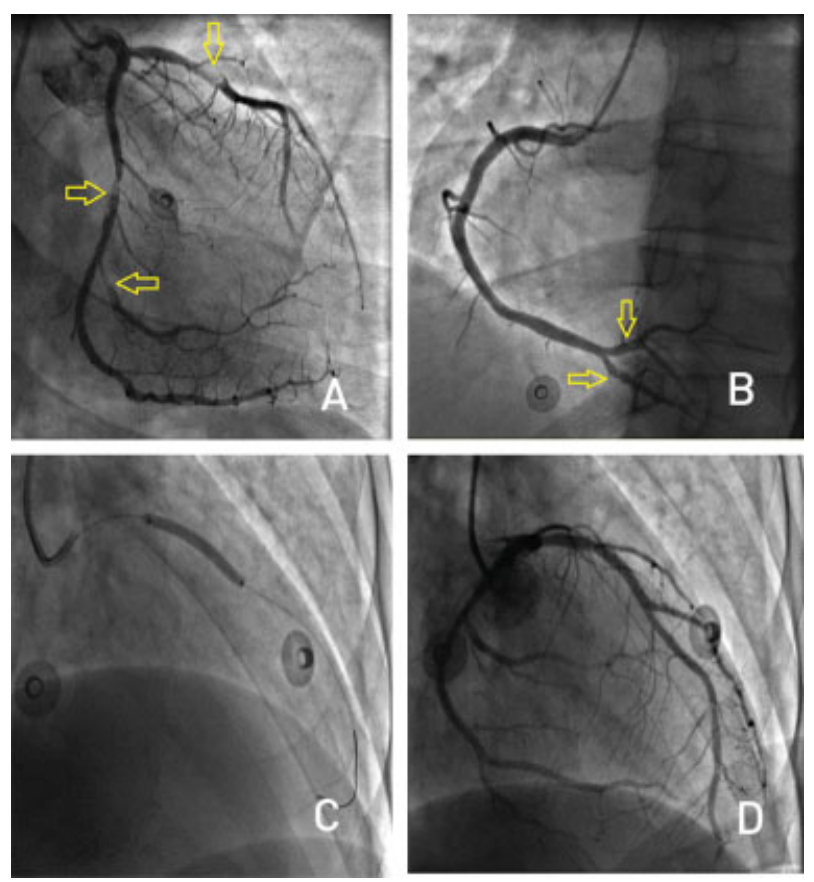

Fig. 2 (A) Thrombus formation in the left anterior descending artery (LAD) and circumflex artery (CXA) in right caudal view. (B) Thrombus formation in the posterior descending artery (PDA) and posterolateral $(\mathrm{PL})$ branches of the right coronary artery (RCA) in left oblique position. (C) Percutaneous coronary intervention of the LAD. (D) Final angiographic result with distal thrombolysis in myocardial infarction (TIMI III) flow after stenting procedure in the LAD. All arrows illustrate the thrombus formations in the coronary arteries. 

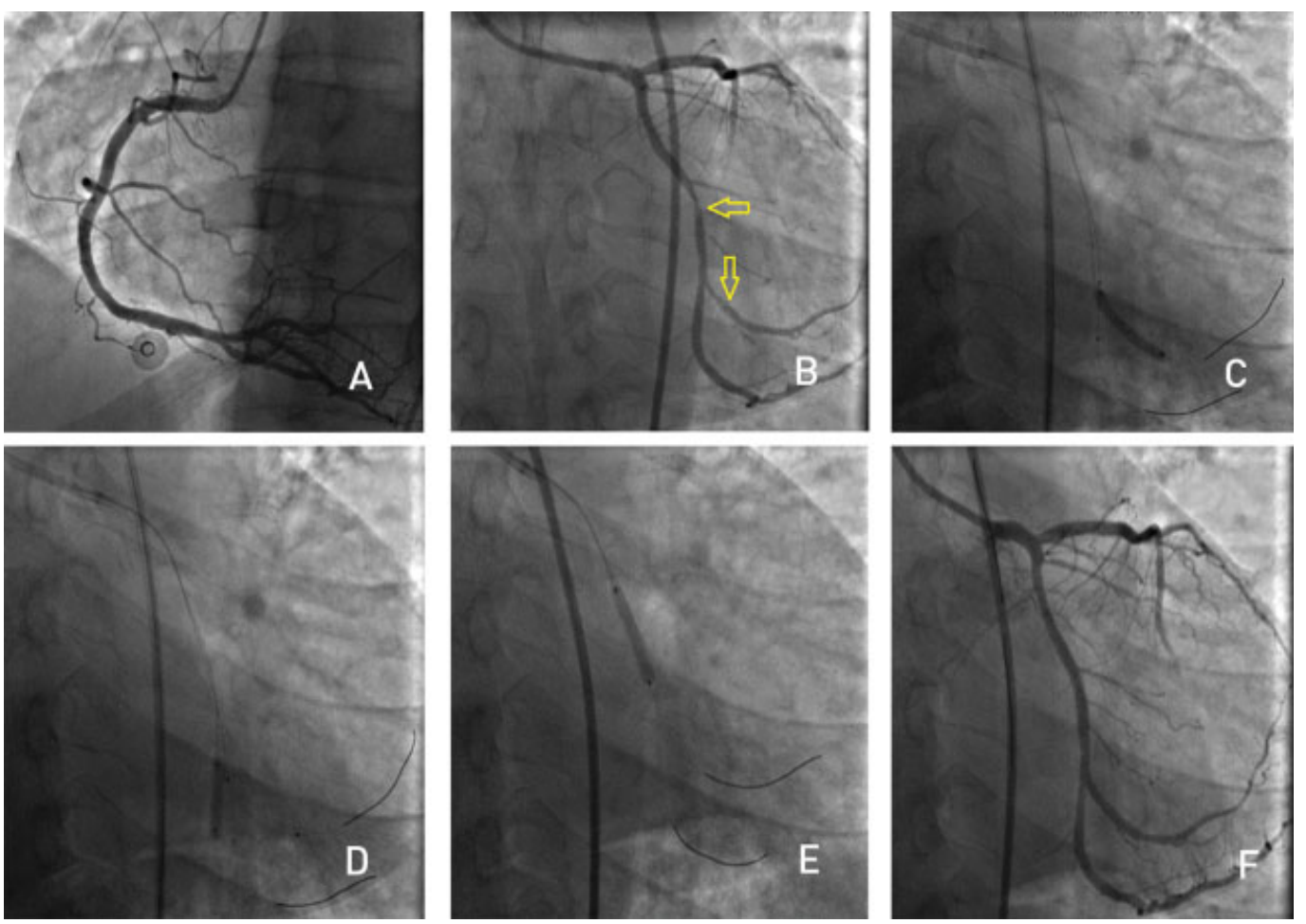

Fig. 3 (A) Angiographic image in left oblique position of the right coronary artery (RCA) after tirofiban administration with thrombolysis in myocardial infarction (TIMI) III flow. (B) Thrombus formation in the circumflex artery (CXA) and obtuse marginal (OM) after tirofiban administration. (C, D) Stenting procedure of the OM and distal CXA with modified flower petal technique. (E) Stenting procedure of the mid-CXA. (F) Final angiographic result of the CXA with distal TIMI III flow in caudal view.

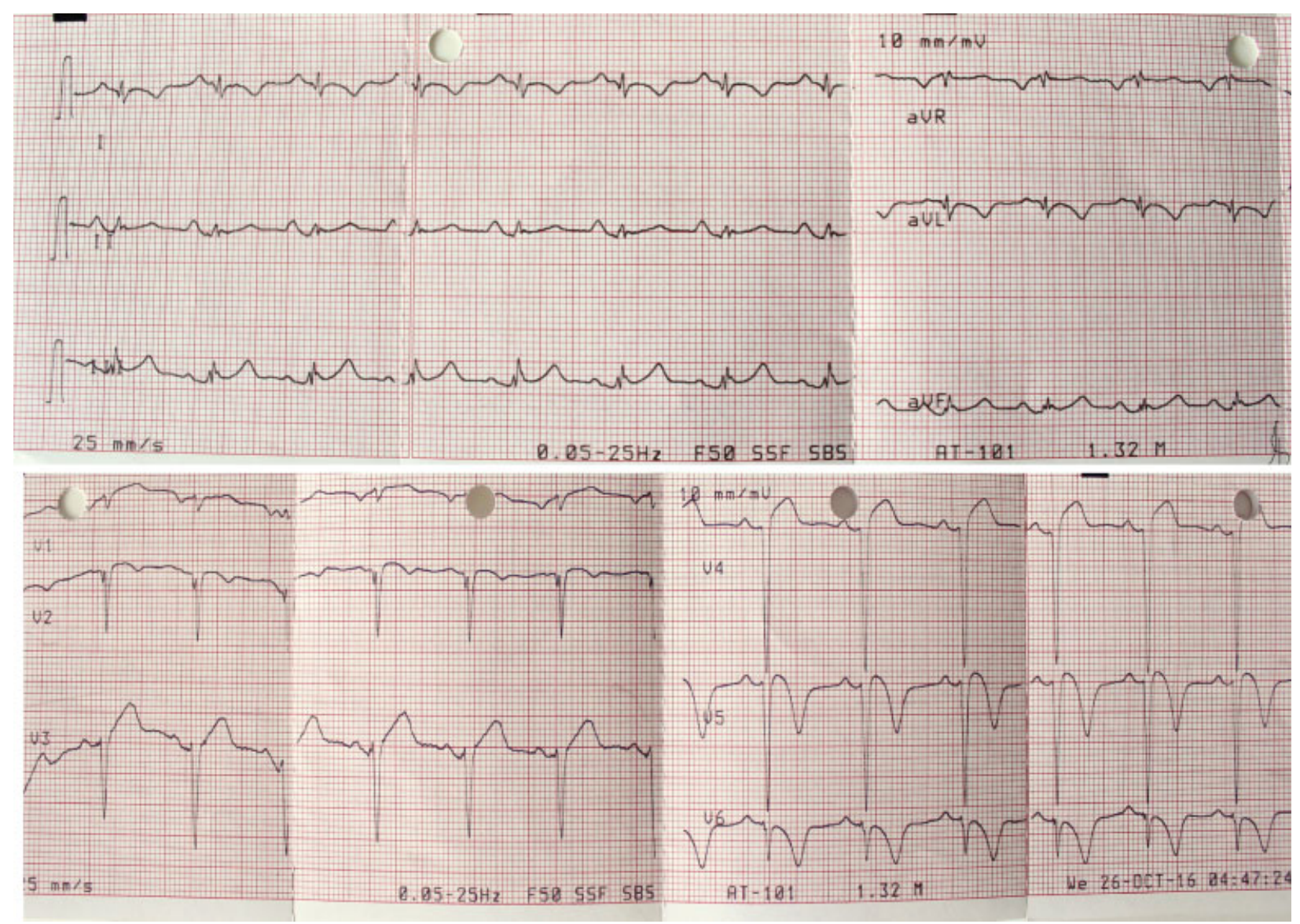

Fig. 4 Subacute electrocardiography (ECG) changes occurred consisting of deeply T wave inversion in particularly lateral leads. 
eluting coronary stent) was implanted with 18 atm in the midCXA (-Fig. 3E) with a final CXA TIMI III flow (-Fig. 3F). After $\mathrm{PCI}$, the patient's hemodynamic parameters were stable. Subacute ECG changes occurred consisting of deeply T wave inversion in particularly lateral leads (-Fig. 4). He was managed with dual antiplatelet therapy: acetylsalicylic acid (100 mg) and ticagrelor (90 mg twice a day) and conservative treatment comprising a $\beta$-blocker (50 mg metoprolol), an angiotensinconverting enzyme (ACE) inhibitor (2.5 $\mathrm{mg}$ ramipril) and statin (40 $\mathrm{mg}$ atorvastatin) therapy. He was discharged from the hospital 5 days after PCI without any symptoms. Hematologic evaluation was recommended for the patient, who had normal platelet levels and had no risk factors for developing a hypercoagulable state except cigarette smoking.

\section{Discussion}

The main pathologic finding of AMI is rupturing of unstable coronary plaques with thrombus formation. This causes subtotal or total occlusion of epicardial coronary arteries. Multivessel coronary artery disease is generally found in STEMI patients. Its prevalence is reported to be from $50 \%$ to $80 \%$., However, multivessel coronary thrombosis is an uncommon finding in acute coronary syndrome patients. More than one coronary artery thrombosis was reported to occur in $1.7 \%$ to $4.8 \%$ of all primary PCIs. In a series of 711 patients reported by Pollak et al, 18 cases of multiple culprit coronary arteries (2.5\%) were found and this generally presented in males (85\%), smokers, and dyslipidemic patients. ${ }^{3}$ Multivessel coronary artery disease patients can present with hemodynamic instability, sudden cardiac death, and mortal ventricular arrhythmias in some cases. ${ }^{4}$ Percutaneous intervention of the culprit lesion is a commonly accepted approach in AMI patients unless hemodynamic instability is involved or a sign of residual ischemia is found. ${ }^{5,6}$ The underlying mechanisms of multivessel coronary thrombosis are not well defined. Some risk factors such as essential thrombocytosis, intravenous cocaine abuse, idiopathic thrombocytopenic purpura, multivessel spasm, hypercoagulability, diabetes mellitus, and smoking were reported in a review of 23 cases. The other possible mechanism is that the first ruptured plaque causes impairment of blood flow in other vessels, increased catecholaminergic activity, and inflammatory processes. ${ }^{7}$ Simultaneous different coronary plaque rupture is a possible mechanism of multivessel coronary thrombosis in acute coronary syndrome patients. This coronary atherosclerotic burden can result in catastrophic outcomes, such as cardiogenic shock and sudden cardiac death. ${ }^{8,9}$ In our case, LAD thrombosis was more impressive, while CXA and RCA were nonflow limiting and this may be the reason why our patient survived. The most common presentation of multivessel coronary thrombosis is both LAD and RCA acute occlusion (49\%). RCA and CXA occlusion (28\%) then follows. ${ }^{10}$

The 12-lead ECG is of importance for determining the culprit coronary artery and for localizing the myocardial infarction area in acute STEMI patients. However, it is not easy to detect the culprit lesion in multivessel coronary thrombosis patients. In our case, the ECG indicated minimal ST-segment elevation in D2-D3 derivations as well as the main elevation in V2-V6 derivations. Supporting this, we performed PCI of the culprit lesion in the LAD with hemodynamic stability. In addition, the patient was treated with glycoprotein IIb/IIIa receptor inhibitor and dual antiplatelet therapy for other thrombotic lesions. We then performed a second intervention in the CXA and OM. The mortality of multivessel coronary thrombosis patients is high due to cardiogenic shock, mortal ventricular arrhythmias, and sudden cardiac death before arriving at the hospital. Early and effective treatment of culprit lesions with complete coronary revascularization (such as with our patient) is of importance for reducing the mortality rate in these patients.

\section{Conclusion}

Simultaneous triple coronary artery thrombosis is a rare presentation of AMI. The mortality rate of these patients is high. Therefore, early diagnosis and effective reperfusion of culprit lesions are key points for successful treatment, as well as medical treatment. Nevertheless, further investigation is needed to determine the best management strategy for multivessel coronary thrombosis patients.

\section{References}

1 Sorajja P, Gersh BJ, Cox DA, et al. Impact of multivessel disease on reperfusion success and clinical outcomes in patients undergoing primary percutaneous coronary intervention for acute myocardial infarction. Eur Heart J 2007;28(14):1709-1716

2 Webb JG, Lowe AM, Sanborn TA, et al; SHOCK Investigators. Percutaneous coronary intervention for cardiogenic shock in the SHOCK trial. J Am Coll Cardiol 2003;42(08):1380-1386

3 Pollak PM, Parikh SV, Kizilgul M, Keeley EC. Multiple culprit arteries in patients with ST segment elevation myocardial infarction referred for primary percutaneous coronary intervention. Am J Cardiol 2009;104(05):619-623

4 Al Suwaidi J, Al-Qahtani A. Multiple coronary artery thrombosis in a 41-year-old male patient presenting with ST-segment elevation myocardial infarction. J Invasive Cardiol 2012;24 (03):E43-E46

5 Lee $\mathrm{CW}$, Lai $\mathrm{CH}$, Lu TM. Double coronary artery thrombosis presenting as acute extensive anterior ST-segment elevation myocardial infarction. J Chin Med Assoc 2013;76(07):407-410

6 Biondi-Zoccai G, Lotrionte M, Sheiban I. Management of multivessel coronary disease after ST-elevation myocardial infarction treated by primary coronary angioplasty. Am Heart J 2010;160 (6, Suppl):S28-S35

7 Kanei Y, Janardhanan R, Fox JT, Gowda RM. Multivessel coronary artery thrombosis. J Invasive Cardiol 2009;21(02):66-68

8 Asakura M, Ueda Y, Yamaguchi O, et al. Extensive development of vulnerable plaques as a pan-coronary process in patients with myocardial infarction: an angioscopic study. J Am Coll Cardiol 2001;37(05):1284-1288

9 Goldstein JA, Demetriou D, Grines CL, Pica M, Shoukfeh M, O'Neill WW. Multiple complex coronary plaques in patients with acute myocardial infarction. N Engl J Med 2000;343(13):915-922

10 Arbustini E, Dal Bello B, Morbini P, et al. Plaque erosion is a major substrate for coronary thrombosis in acute myocardial infarction. Heart 1999;82(03):269-272 